Proc. Indian Acad. Sci., Vol. C 2, Part 2, May 1979, pp. 243-261, (C) Printed in India.

\title{
Systems analysis for planning of air fleets and maintenance facilities
}

\author{
V V S SARMA*, K RAMCHAND ${ }^{\dagger}$ and A K RAO** \\ Indian Institute of Science, Bangalore 560012 \\ *School of Automation \\ **Department of Aeronautical Engineering \\ $\nmid$ Present Address: CSDO, Air Force Station, Chakeri, Kanpur 208008
}

MS received 23 March 1978; revised 21 October 1978

\begin{abstract}
The high cost and extraordinary demands made on sophisticated air defence systems, pose hard challenges to the managers and engineers who plan the operation and maintenance of such systems. This paper presents a study aimed at developing simulation and systems analysis techniques for the effective planning and efficient operation of small fleets of aircraft, typical of the air force of a developing country. We consider an important aspect of fleet management: the problem of resource allocation for achieving prescribed operational effectiveness of the fleet. At this stage, we consider a single flying-base, where the operationally ready aircraft are stationed, and a repair-depot, where the planes are overhauled. An important measure of operational effectiveness is ' availability', which may be defined as the expected fraction of the fleet fit for use at a given instant. The tour of aircraft in a flying-base, repair-depot system through a cycle of 'operationally ready' and 'scheduled overhaul ' phases is represented first by a deterministic flow process and then by a cyclic queuing process. Initially the steady-state availability at the fiying-base is computed under the assumptions of Poisson arrivals, exponential service times and an equivalent singleserver repair-depot. This analysis also brings out the effect of fleet size on availability. It defines a ' small' fleet essentially in terms of the important 'traffic' parameter of service rate/maximum arrival rate.

A simulation model of the system has been developed using GPSS to study sensitivity to distributional assumptions, to validate the principal assumptions of the analytical model such as the single-server assumption and to obtain confidence intervals for the statistical parameters of interest.
\end{abstract}

Keywords. Systems analysis; queuing models; air fleet planning; maintenance; GPSS simulation.

\section{Introduction}

This paper reports a systems engineering study useful for the effective planning and efficient operation of small fleets of aircraft, typical of the air force of a developing country. Such studies, it is believed, will guide engineering and management decisions and lead to more efficient allocation of the limited available resources between several competing components. Such studies are particularly important in an era of the essentially level, or declining, purchasing power of military budgets against the rapidly increasing cost and complexity of weapon systems.

The management problems of an air force are complex, consisting typically of problems in planning, operations and logistics. For example, consider the particular

A list of symbols appears at the end of the paper. 
case of the Indian Air Force (IAF). The IAF is one of the large organisations of the country, with an annual budget of several hundreds of crores (billions) of rupees, employing a few lakhs of personnel and controlling thousands of advanced military weapons and about a hundred military bases and operating locations. The air force is also the principal customer for the country's aeronautical industry and allied research and development $(\mathrm{R} \& \mathrm{D})$ activities.

\subsection{Planning problems}

The support planning for a new aircraft fleet begins almost at the time the decision is made to accept a proven design and develop a new air fleet. It may be 3 to 5 years before the fleet becomes operational. The planning agency estimates the support requirements such as repair-depot facilities, personnel, equipment and spares. Planning also involves the study and analysis of $R \& D$ policies, the economics of technological change and growth, and strategies for ačquiring fleet and weapon systems.

\subsection{Operations problems}

When operational organisations such as flying-bases and repair-depots start to function, the first indication of their actual operating capability emerges. But at this time there is very little opportunity for improvement of the aircraft, say, in terms of reliability and maintainability. One of the major operations problems of a flyingbase is scheduling. Scheduling involves two major components: (1) operations scheduling, for planning of sorties and training of crews, and (2) maintenance scheduling, which involves selection of aircraft for each training mission and the scheduling of maintenance of vario us levels, depending on the flight hours logged by each aircraft.

\subsection{Logistics problems}

The major logistics problems arising in fleet management deal with maintenance and spares requirements. The main problem here is to find out for how long a multimillion rupee aircraft is not fit for use, when needed. Some of the many reasons for this to happen are (1) the reliability and maintainability of an aircraft, which are important parameters determining an aircraft's 'down time', (ii) scheduled or unscheduled maintenance, (iii) inadequacy of the repair-depot, (iv) repair crew not available, (v) spares not available, and (vi) deterioration due to actions like cannibalisation. At the outset, we should recognise that the problems of a military organisation share much in common with those of industrial and commercial organisations except that the defence system suffers more from the stresses of large scale organisation and geography, the rapidly changing weapons technology and the need for flexibility to meet the distinctly different demands made under conditions of war and peace. The problems of military management have posed a difficult challenge to management science and operations research. Institutions like the RAND Corporation have been actively engaged in studying these problems since 1950 in the context of US military organisations. Some of their unclassified research efforts in this direction have been well documented in management science literature and RAND reports (Geisler 1960; Donaldson 1971; Dade 1973). 


\section{Background for the present work}

An air force operates several types of aircraft fleets such as combat aircraft, transport aircraft, helicopters, trainer aircraft and bombers. The present work is primarily motivated by our interest in the problems of the trainer aircraft of the IAF. India currently uses four types of trainers, namely the HT-2, HJT-16, HPT-32 and TS- 11. The HT-2 is a piston-engined aircraft indigenously produced and has been in use since 20 years; it is in the process of being replaced. It has several flying-bases (training centres) and a repair-depot for overhauls. The HJT-16 is a jet-trainer and is the backbone of the current pilot training activities. This aircraft was designed and is produced indigenously and is overhauled by the air force; it is expected to be in service till the 1990s. The HPT-32 is a new type of Indian designed piston-engined aircraft, which is to be produced indigenously; and it is proposed to be introduced into service in the next few years. While the three types of aircraft mentioned above are indigenously produced, several sub-systems, most notably the engines, are imported. Normally unanticipated situations of demand, shortage and delay in indigenous production require interim measures such as outright import of a fleet. The TS-11 is a Polish jet trainer introduced under such conditions and its fleet will be in service for the next decade.

Each individual aircraft is a complex system. There is no worthwhile engineering commonality between any two types of aircraft. In view of this it would be normal

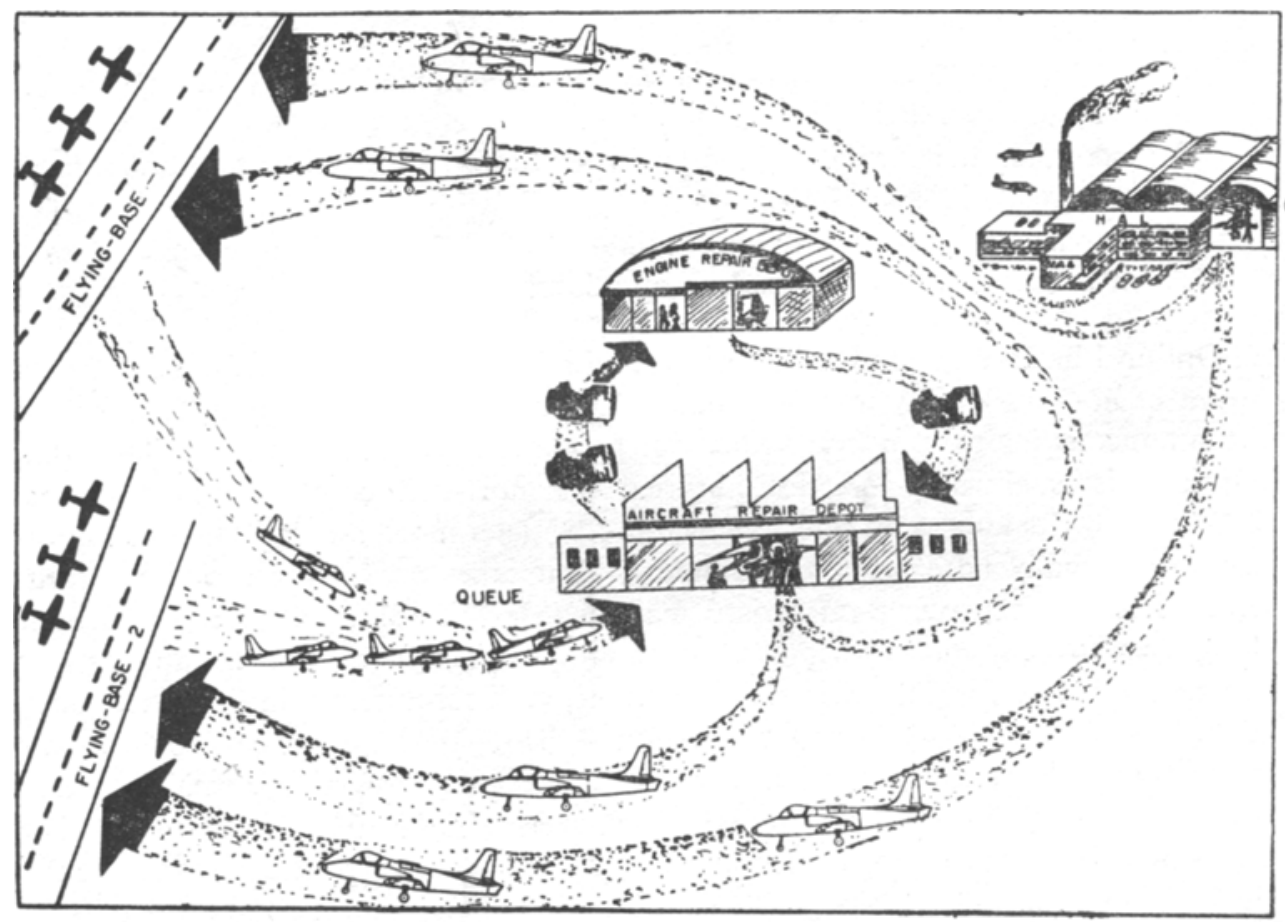

Figure 1. Iconogram of the cyclic queuing process arising in aircraft fleet operations. 
practice to have separate repair facilities and distinct flying bases for each type. So we may assume that the problem is one of management of small (say between 25200) fleets, each of one type of aircraft. Each fleet has its own planning, operations and logistics problems. As an example, let us consider the operation of the fleet of HJT-16s. Figure 1 shows the iconogram of the operations of a fleet of HJT-16. The two flying-bases operating these aircraft have to train pilots and flying instructors. The problem of the flying-base commanders is the effective scheduling of aircraft for training and maintenance purposes. For this, they need a minimum number of aircraft at the flying-bases.

A measure of the effectiveness of the system represented in figure 1 is the number of aircraft fit to fly at any given instant at the flying-bases. Normally a trainer aircraft is used for a fixed flying time, say $600-1000 \mathrm{hr}$, and then sent for a scheduled overhaul to a repair-depot. The prescribed flight time is normally logged over 2-3 calendar years. While this scheduling problem is of some interest, the major problem that arises in the operation is the progressive reduction in available aircraft at the flying-base because of increased congestion at the repair-depot. An effective solution to this problem lies beyond the flying-base commander's control. So if the available aircraft consistently falls below a stipulated number, several options are open to remedy the situation, but all of them need decisions at a higher level. Some of the options are:

(i) having a maintenance reserve of aircraft (by initial acquisition of extra aircraft),

(ii) augmenting the fleet by acquiring additional aircraft (as the situation develops),

(iii) modifying preventive maintenance policies (extending the overhaul life say, from 600 to $700 \mathrm{hr}$ ),

(iv) increasing the capacity of the repair-depots, by investing in additional facilities, men and spares, and

(v) increasing the reliability and maintainability of individual aircraft by extra investments in design, research and development.

Option 1 has been often tried in practice with limited success; it only postpones the event of non-availability to a later date. Option 2 is a short-term cure, leading to instantaneous increase in availability but greater congestion results at a later date. Option 3 is usually an ad hoc decision tried as a short-term cure and is based on past experience. Its impact on safety etc. cannot be determined and therefore this option cannot normally be exercised indefinitely, as it takes considerable time to gather fresh data and experience on revised maintenance schedules. Option 4 is a better long-term strategy but conscious effort should be made even at the planning stage because of long pipelines for spares and heavy unproductive initial investments. Other short-term cures to reduce congestion at the repair-depot are cannibalisation of aircraft for spare parts (which has its own evil effects), transferring of spares between production and repair organisations, lower levels of utilisation of aircraft, and increasing throughput of repair-depots by ad hoc measures. In fact, option 4 interlinks operational and logistics problems. Option 5 is a long-term option which should be incorporated at the planning stage itself. In this procedure, there is a degree of uncertainty about cost-benefit ratios and time scales. Thus the fleet management 
problem is essentially a resource allocation problem between the options of choice of fleet size, repair-depot capacity planning, inventory management of spares, and finally design, research and development efforts for improved reliability and maintainability. It may be noted that all the five options mentioned above are not open to every type of aircraft. Thus each of our fleets is in its own peculiar situation. Management of each to increase operational effectiveness needs a separate systems study with its own models, criteria, constraints and solutions

At this point, it is appropriate to survey other work on problems of aircraft fleet management available in open literature. LaValle \& Stoller (1962) of RAND give a brief outline of a field study of the effectiveness of an interceptor squadron in terms of reliability and maintainability. In particular they used field data to establish the validity of the Poisson distribution for characterising both the failures generated by system operation and the time required for repair. They used several measures for evaluating the effectiveness such as (1) turn around time, (2) abort rate, (3) fraction of aircraft that have in-flight malfunctions, (4) time-out-of-commission of a sortie, (5) sortie rate, (6) man hours for each maintenance job, and (7) time an aircraft is awaiting parts, equipment or personnel. All these quantities were obtained by elaborate controlled field tests when the squadron flew 844 sorties totalling $703 \mathrm{hr} 45 \mathrm{~min}$ of aircraft flight time. While the use of field data from such controlled tests is essential for on-line estimation and correction of deviations from planned paths, such data are not usually available at the planning stage. Wood (1971) uses computer simulation as a tool to determine the cost effectiveness of different levels of reliability and maintainability for a training aircraft in the RAF. His results give the number of aircraft required to produce a mean daily output of 65 flying hours at three levels of reliability (characterised by three defect rates of $0.35,0.70,1.05$ defects per flying hour and three levels of maintainability $(5,10,15$ man hours per defect). Moe (1976) considers the small fleet (24 aircraft) problem in the context of availability on an aircraft carrier and again uses a simulation model, the Avionics Logistics Effects (ALE) model, to evaluate potential benefits of reliability and maintainability improvements that can be incorporated into the AWG-9 weapon control system on the F-14 aircraft.

The studies of both Wood and Moe deal with flight line servicing, which is the responsibility of the flying-base commander; they do not consider repair-depot overhauls. Chittaranjan (1974) describes the current maintenance practice in the IAF. First-line servicing includes routine check-up of aircraft before and after flying by checking against a maintenance checklist. Second-line servicing involves minor repairs and replacement of components. Third-line servicing is for unscheduled maintenance for damaged aircraft. The fourth-line service is for scheduled overhauls at a repair-depot. In the present study, we consider fourth-line servicing, as this is the most important aspect of maintenance planning for resource allocation and long range planning.

This paper is organised as follows. In $\S 3$, we consider the flying-base, repairdepot system in steady state. In $\S 4$, we relax the steady-state assumption and consider dynamic modelling. $\$ 5$ deals with a GPSS simulation of the system and provides an alternative and complementary approach to the problems considered by $\S \S$ 3 and 4 . The whole study illustrates an application of the emerging methodology of systems engineering. 


\section{Steady state queuing models and static optimisation}

In this section, we discuss the problem of estimating the operational effectiveness of a fleet of trainer aircraft in the steady state. For simplicity in analysis, we assume that there is a single flying-base, where the operationally ready aircraft are stationed for pilot training and a repair-depot where the planes are overhauled. The value of the flying-base, repair-depot system is its productivity in terms of its total flight hours per annum and its operational effectiveness, one important measure of which is availability. We define availability as the average fraction of aircraft fit (available) for use at a given instant. The remaining (unavailable) aircraft are at the repair-depot either under scheduled overhaul or in a queue awaiting repair. Flight line and other unscheduled maintenance is assumed to have a negligible effect in these availability calculations. A trainer aircraft is normally used for a fixed fiying time, say 600 $1000 \mathrm{hr}$, then sent for scheduled overhaul. This flight time is normally logged over a tour length of 2 to 3 calendar years. The non-availability of aircraft is due to the non-zero repair time (3-4 months). Repair delays are acute for small fleets since any deviation from the schedule of arrivals at the repair-depot or at the flying-base seriously affects the availability.

Rose (1973) considers a version of the short-term problem and applies the results to the availability of a fleet of 300 US Navy F-4 aircraft. He conlcudes that it is more economical to cut down the delay in the repair-depot by investing in additional inventory of spares than to buy additional aircraft. This conclusion is of course based on a cost structure which favours buying of spares. Rose suggests that better results could be obtained with the aid of queuing models. In this section, we consider a deterministic model and a cyclic queue model for this problem. This helps to relate the parameters of interest such as flight-time, scheduled arrival rate, repair time of aircraft and repair rate at the repair-depot.

\subsection{A deterministic model for availability}

Consider the flying-base, repair-depot model shown in figure 2. To study the normal operation of the system and to understand the relationship between the various parameters, it is necessary to visualise a flow model as shown in figure 3 . The aircraft

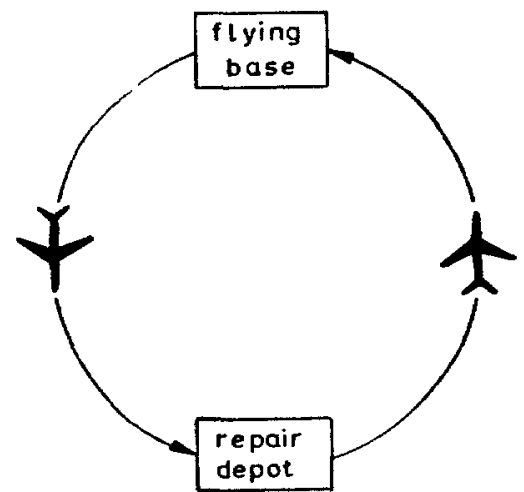

Figure 2. Single fiying-base, repair-depot model, 


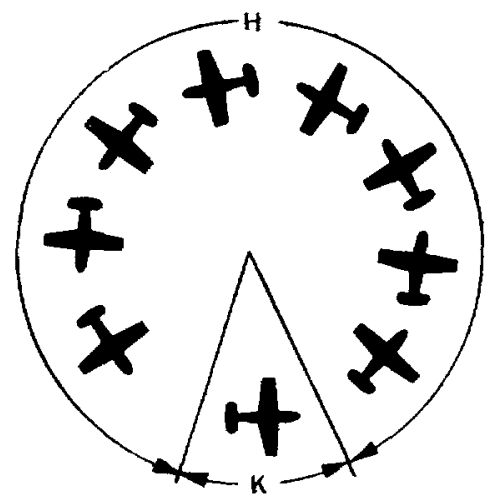

Figure 3. Cyclic flow mode].

move along the circle in steady state with uniform speed. The total number of aircraft are spaced at uniform intervals on the circumference of the circle. The equations for the deterministic model can now be easily written as (Sarma et al 1977)

$$
T=H+K ; \lambda=1 / T ; \mu=N_{a} / T ; A_{1}=H / T ; F=\mu f
$$

From equation (1), we get

$$
N_{B}=N_{a} H / T \text { and } N_{D}=N_{a} K / T \text {. }
$$

In this deterministic model, the productive flight time per calendar year $F$ depends only on $\mu$, and though the availability $A_{1}$ increases slightly with decrease in $\mu$, there is a big reduction in $F$, if a queue is avoided. Avoiding a queue by increasing $H$ is thus not a useful solution.

\subsection{A cyclic queue model}

In practice, the scheduled maintenance of aircraft as suggested by the deterministic model of $\S 3.1$, cannot be achieved. There can be unexpected delays at both the entry and exit points of the repair-depot. To obtain more realistic availability figures, it is worth considering the arrivals and services as random processes. An examination of figure 2 shows that the combined process is a cyclic queue system.

The steady state probability $P_{n}$ of finding $n$ aircraft in the repair-depot is given by (Sarma et al 1977)

$$
P_{n}=\frac{\left[N_{s} ! /\left(N_{s}-n\right) !\right](\lambda / \mu)^{n}}{\sum_{i=0}^{N_{s}}\left\{\left[N_{s} ! /\left(N_{s}-i\right) !\right](\lambda / \mu)^{i}\right\}},
$$

where $\quad N_{s}=N_{a}(H / T)+1$.

The expected number of aircraft in the repair-depot in the steady-state is given by

$$
N_{D}=\sum_{n=0}^{N_{s}} n P_{n}
$$


The steady-state expected number of aircraft available at the flying base is given by

$$
N_{B}=N_{s}-N_{D}
$$

The availability is

$$
A_{\mathbf{2}}=N_{B} / N_{a}
$$

where $\quad N_{a}=\left(N_{s}-1\right)(H+K) / H$ rounded to the nearest integer.

Table 1 shows the results obtained for small fleet sizes. A significant observation from the availability figures is that for the fleet sizes considered, the steady state availability is directly proportional to the repair capability for small values of $\mu$. For example, in the case of fleet sizes considered in table 1 , for $\mu=10, N_{B}$ is 10 and for $\mu=25, N_{B}$ is 25 . At these low values of $\mu$ relative to fleet size, investments in additional aircraft may only temporarily increase the availability and may not have any effect on the long term (steady state) availability. On the other hand, for a relatively high value of $\mu$, an increase in fleet size improves availability. Therefore, it may be concluded on the basis of steady state models that while setting up the repair capability one must invest in a fairly large repair capability to meet the possibility of increasing demands of the flying base in terms of availability over the entire planning horizon.

\subsection{Time spent by an aircraft in the repair-depot}

The time spent by an aircraft in the repair-depot is another parameter of interest in the design of service facilities. In the case of cyclic queues, the well known Little's

\begin{tabular}{|c|c|c|c|c|c|}
\hline$N_{s}$ & $N_{a}$ & $\mu / \lambda$ & $N_{B}$ & $N_{D}$ & $\begin{array}{l}\text { Percentage } \\
\text { availability } \\
A_{2}=N_{B} / N_{a}\end{array}$ \\
\hline \multirow[t]{4}{*}{25} & 27 & 10 & 10 & 15 & 37 \\
\hline & & 25 & 21 & 4 & 78 \\
\hline & & 35 & 22 & 3 & 81 \\
\hline & & 60 & 23 & 2 & 85 \\
\hline \multirow[t]{4}{*}{35} & 38 & 10 & 10 & 25 & 26 \\
\hline & & 25 & 25 & 10 & 66 \\
\hline & & 35 & 30 & 5 & 79 \\
\hline & & 60 & 33 & 2 & 87 \\
\hline \multirow[t]{4}{*}{41} & 45 & 10 & 10 & 31 & 22 \\
\hline & & 25 & 25 & 16 & 55 \\
\hline & & 35 & 33 & 8 & 73 \\
\hline & & 60 & 38 & 3 & 84 \\
\hline \multirow[t]{5}{*}{45} & 50 & 10 & 10 & 35 & 20 \\
\hline & & 25 & 25 & 20 & 50 \\
\hline & & 35 & 35 & 10 & 70 \\
\hline & & 60 & 42 & 3 & 84 \\
\hline & & 100 & 44 & 1 & 88 \\
\hline
\end{tabular}

Table 1. Availability at the flying-base. $H=2$ years, $K=3$ months, $\lambda=1$ arrival per year 
formula, $N_{D}=\lambda W$ (Little 1961) relating queue length and time spent in the system is not valid. This is a major difference between cyclic and infinite queuing systems, and it arises from the finite source population of cyclic queues. The expression for waiting time $W$ of a cyclic queue is given by

$$
W=\mu^{-1}\left(N_{s}+1\right)\left(1-P N_{s}\right)-\lambda^{-1}\left(1-P_{0}\right)
$$

Table 2 shows the results obtained for various fleet sizes, and $(\mu / \lambda)$ values for the expected time spent by an aircraft in the repair-depot.

\subsection{Finite queue model for spares}

The steady-state availability computations of $\S 3.2$ show that the repair-depot capability is a significant factor contributing to the availability. In practice, 'stock-out' of critical spares effectively reduces $\mu$ (Gross 1975). In air fleet management, the number of repairable spares constitute a smaller percentage than consumable spares but their total budgetary demands are much higher. So the repairable spares have a more pronounced effect on the availability than the consumable spares. This can be seen from the fact that gun sight, fuel pump, generator set and engine are repairable items. Figure 4 schematically represents the interaction of the maintenance and

Table 2. Steady-state waiting time in days for various effective fleet sizes. $\quad(\lambda=1)$

\begin{tabular}{ccrrrrr}
\hline$\mu / \lambda$ & \multicolumn{6}{c}{ Effective fleet size $-N_{s}$} \\
\cline { 2 - 7 } & 25 & 35 & 41 & 45 & 75 & 100 \\
\hline 100 & 4 & 5 & 5 & 5 & 10 & 26 \\
75 & 6 & 7 & 8 & 9 & 30 & 104 \\
60 & 8 & 11 & 14 & 16 & 82 & 208 \\
50 & 11 & 17 & 22 & 28 & 156 & 306 \\
$37 \cdot 5$ & 20 & 37 & 57 & 78 & 308 & 508 \\
$33 \cdot 3$ & 26 & 54 & 87 & 117 & 384 & 608 \\
30 & 34 & 76 & 121 & 161 & 460 & 710 \\
\hline
\end{tabular}

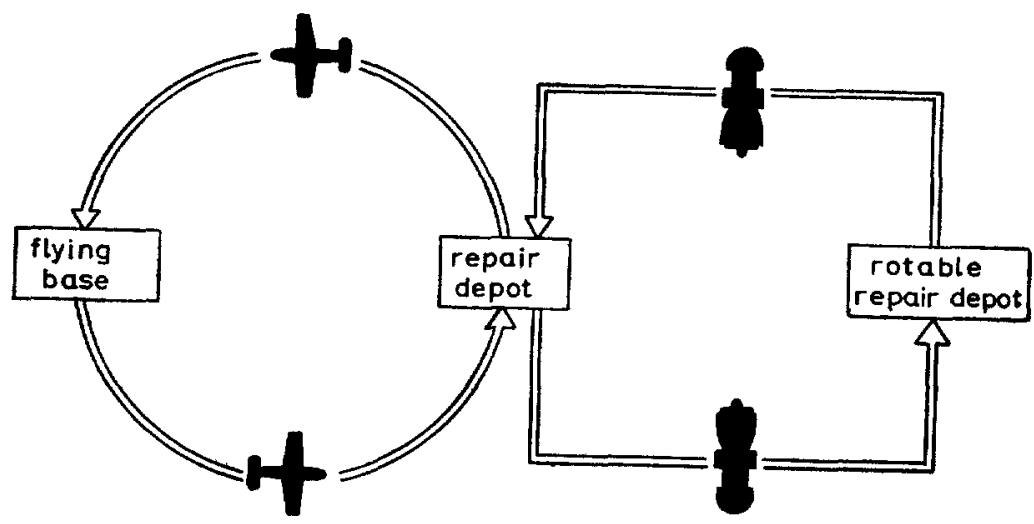

Figure 4. Model of interaction between flying-base, repair-depot and one type of repairable spares. 
repairable spares models. For analysis, let us assume that there is one type of such a critical spare, say, the engine. Let us assume that the aircraft are single-engined. Let $\mu_{R}$ be the service rate of the spare repair shop. Further, if we assume that every scheduled maintenance of an aircraft needs an engine replacement, the demand rate of the engine is $\mu$. If $M$ is the total number of spare engines available, birth and death equations governing the probabilities $q_{n}(t), n=0, \ldots, M$ where $q_{n}(t)$ denotes the probability that there are $n$ engines at the rotable repair shop, can be easily written as (Koenigsberg 1960),

$$
\begin{aligned}
& q_{0}^{\prime}(t)=-\mu q_{0}^{(t)}+\mu_{R} q_{1}^{(t)} \\
& q_{n}^{\prime}(t)=\mu_{R} q_{n-1}(t)-\left(\mu+\mu_{R}\right) q_{n}(t)+\mu q_{n+1}(t), \\
& q_{M}^{\prime}(t)=\mu q_{M-1}(t)-\mu_{R} q_{M}(t)
\end{aligned}
$$

Here we are only interested in the steady-state probability $q_{M}$ which corresponds to stock-out. This is given by

$$
q_{M}=\left(\mu / \mu_{R}\right)^{M} /\left\{\sum_{i=0}^{M}\left(\mu / \mu_{R}\right)^{i}\right\}
$$

The probability that a spare engine is available when demanded is $\left(1-q_{M}\right)$. The effective repair-depot capability $\bar{\mu}$ is given by

$$
\bar{\mu}=\mu\left(1-q_{M}\right)
$$

\section{Transient analysis and dynamic optimisation}

In this section, we move on to the dynamic analysis of the flow of aircraft in a flyingbase, repair-depot combination. Two of the main criticisms against $M / M / 1$ models in steady-state are: first, that the assumption of Poisson arrivals and negative exponential repair times may be as much in error as the assumption of constant tour length and service time of the deterministic model, and secondly, the assumption that the system settles down to its steady-state may not be valid in finite horizon problems. The first objection has been discussed by several authors (Koopman 1972; Maher \& Cabrera 1975). The second objection about the steady-state assumption is more serious. Narayan Bhat (1969) has observed that in many realistic queuing situations, it takes a considerable portion of the operating time for the steady-state situation to be achieved. In the case of an HJT-16 fleet, the fleet strength and repair capability is modified every year. It is obvious that transient analysis is to be carried out, to obtain the availability profile in these cases over the planning horizon. In general, this is true of the planning of any new air fleet, like that of the HPT-32. The life span of an aircraft is about 20 years. If the proposed final fleet strength is 200 aircraft, building up the fleet will take at least 5-10 years and the repair-depot will also reach its planned maximum capacity in about the same time. So, for optimum allocation of resources between acquiring new aircraft and augmentation of repair facilities in the build-up stage, transient analysis is necessary. 


\subsection{Differential equation for queue size}

If $N_{s}$ is the effective fleet size, $\lambda$ is the mean arrival rate of an aircraft at the depot in years, $\mu$ is the mean repair rate of the depot, in aircraft repaired per year, and $P_{n}(t)$ the state probabilities describing the number $n$ of aircraft in the queue at any time $t$, the differential equations governing $P_{n}(t)$ may be written as

$$
\begin{aligned}
& P_{0}^{\prime}(t)=-N_{s} \lambda P_{0}(t)+\mu P_{1}(t), \\
& P_{0}^{\prime}(t)=-\left[\left(N_{s}-n\right) \lambda+\mu\right] P_{n}(t)+\mu P_{n+1}(t)+\left(N_{s}-n+1\right) \lambda P_{n-1}(t),
\end{aligned}
$$

with initial conditions $P_{0}(0)=1, P_{n}(0)=0, n=1,2, \ldots N_{s}$.

But, if the required parameter is only availability of the aircraft at base, it would be sufficient if the number in the queue at a given time

$$
Q(t)=\sum_{n=0}^{N_{s}} n P_{n}(t)
$$

is evaluated. Differentiating (12) and substituting the values of $P_{n}^{\prime}(t)$ from (11) and after simplification, we obtain

$$
Q^{\prime}(t)=-\lambda Q(t)+N_{s} \lambda-\mu\left(1-P_{0}(t)\right), Q(0)=0 .
$$

Equation (13) is a simple first order differential equation provided $P_{0}(t)$ can be evaluated or approximated without solving (11). In the aircraft fleet problems which we are considering, $\lambda$ is about $0 \cdot 2$ to 1 arrivals per year, $\mu$ is of the order of 10 to 100 aircraft per year and $N_{s}$ is about 10 to 100 aircraft. An examination of (11) and (13) reveals the following:

(i) at $t=0, Q^{\prime}(t)=+N_{s} \lambda$,

(ii) at $t=0, P_{0}^{\prime}(t)=-N_{s} \lambda$ and $P_{1}^{\prime}(t)=N_{s} \lambda$.

Initially the queue builds up fast and $P_{0}(t)$ rapidly falls to a low value. The steadystate value of $P_{0}=0$ for the range of numerical values of interest of parameters $N_{s}$, $\mu$ and $\lambda$. For this case, (13) may be simplified to

$$
Q^{\prime}(t)=-\lambda Q(t)+N_{s} \lambda-\mu ; Q(0)=0,
$$

i.e.

$$
Q^{\prime}(t)=\left(N_{s}-Q(t)\right) \lambda-\mu ; Q(0)=0
$$

In contrast to (13), (15) can be readily solved. It may be easily seen that (15) for queue build-up is valid only when $\mathbf{N}_{s} \lambda>\mu$.

\subsection{Remarks}

(i) While (15) is an approximation for the number in the queue in the case of Poisson arrivals and exponential service, it is an exact equation for the deterministic

Pro. (C) -7 
queue. This can be derived by the fluid-flow approximation where the variables are assumed to be continuous rather than discrete. When this is done the conservation law is the differential equation (15), relating the number of aircraft in the system to the user arrival rate $\left(N_{s}-Q\right) \lambda$ and the throughput $\mu$ giving $Q^{\prime}(t)=\left(N_{s}-Q\right) \lambda-\mu$. Agnew (1976) uses a similar argument in his dynamic analysis of congestion-prone systems with infinite population.

(ii) The validity of this approximation again brings to light the relative insensitivity of parameters such as queue size to the distributional assumptions (the two extremes of the deterministic case and the purely random case) (Koopman 1972). The solution for $(15)$ is

$$
Q(t)=\left(N_{s}-\mu / \lambda\right)(1-\exp (-\lambda t)),
$$

and

$$
Q_{s s}=\left(N_{s}-\mu / \lambda\right)
$$

Equation (16) gives the build-up of the queue. The approximate value of the steadystate queue size is given by (17). Table 3 gives the exact and approximate values of the number of aircraft in the steady queue at the repair-depot for several values of $N_{s}$ and $\mu$. As can be anticipated from (13) and (15), for larger values of $\mu$ (as $\mu \rightarrow N_{s} \lambda$ ), results obtained from the approximate expression are optimistic because $\mu P_{0}(t)$ is neglected on the right hand side of (14). On the other hand the exact expression for $Q_{s s}$, as seen from (14) is

$$
Q_{s s}=N_{s}-(\mu / \lambda)\left(1-P_{0}\right)
$$

\subsection{Fleet size affects availability}

Equations (17) and (18) characterise the effects of the 'smallness' of a fleet. As seen from (18), the percentage availability is $100\left(\mu / \lambda N_{s}\right)$ and is shown in figure 5.

Table 3. Comparison of the exact and approximate values of the number of aircraft at the repair-depot in steady-state. $\lambda=1$

\begin{tabular}{lccc}
\hline \multirow{2}{*}{$N_{s}$} & & \multicolumn{2}{c}{$\begin{array}{c}\text { Number in the queue at the } \\
\text { repair-depot }\end{array}$} \\
\cline { 3 - 4 } & & $\begin{array}{c}\text { Exact } \\
\text { (equation (4)) }\end{array}$ & $\begin{array}{c}\text { Approximate } \\
\text { (equation (17)) }\end{array}$ \\
\hline \multirow{2}{*}{25} & 10 & 15 & 16 \\
& 15 & 10 & 10 \\
& 20 & 6 & 5 \\
& 25 & 4 & 0 \\
& 10 & 35 & 35 \\
& 15 & 30 & 30 \\
& 20 & 25 & 25 \\
& 30 & 20 & 20 \\
& 30 & 20 & 15 \\
& 35 & 11 & 10 \\
& 40 & 8 & 5 \\
& 45 & 6 & 0 \\
\hline
\end{tabular}




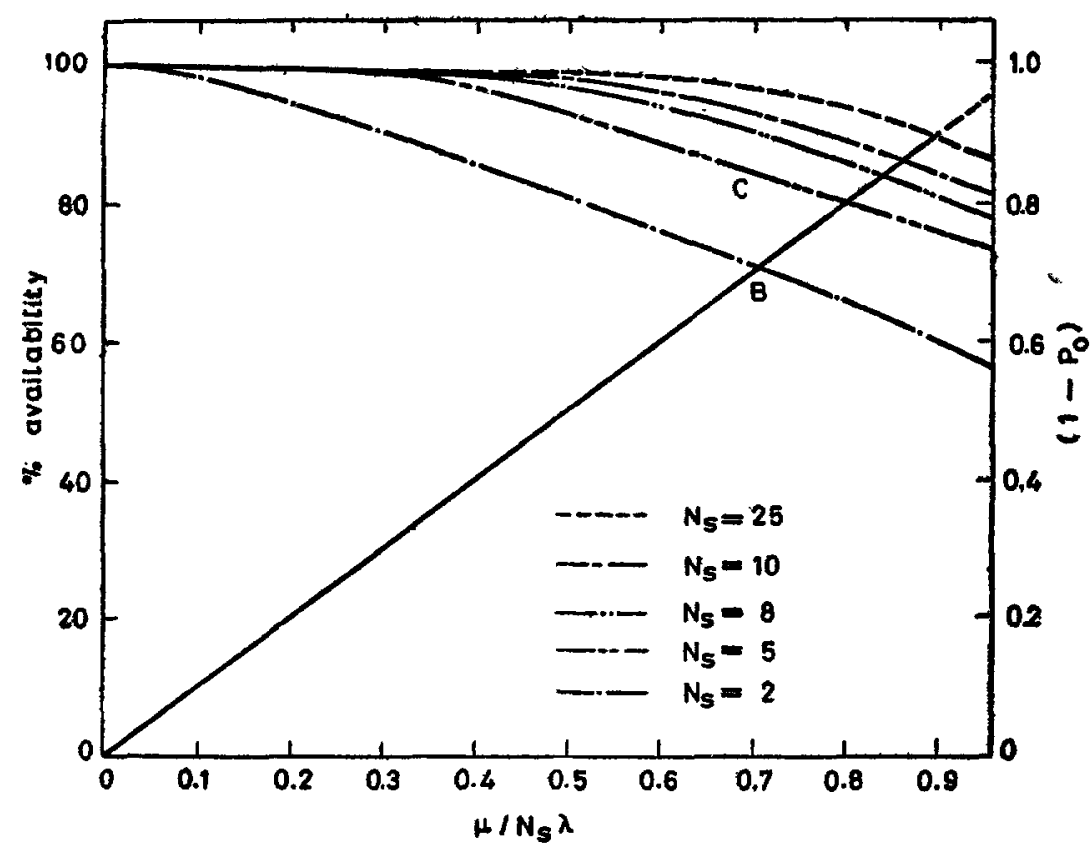

Figure 5. Percentage fleet availability for all fleet sizes with correction for fleet size.

$\left(1-P_{0}\right)$ values for various fleet sizes are displayed on the same graph for the range 0.1 to 0.9 of $\left(\mu / \lambda N_{s}\right)$ values. Figure 5 can be used to generate the availability curves for any fleet size.

The non-dimensional factor $\left(\mu / \lambda N_{s}\right)$ determines a quick approximation for the percentage availability of the fleet. In figure 5 , the abscissa at any given value of $\left(\mu / \lambda N_{s}\right)$ gives this approximate value, while its product with the ordinates of the $\left(1-P_{0}\right)$ curves gives the exact availability for specific fleet sizes. The need for the correction increases with increasing $\left(\mu / N_{s} \lambda\right)$ and decreasing fleet size.

Given $\left(\mu / \lambda N_{s}\right)=0.7$, the approximate availability for any fleet size is $70 \%$. If $N_{s}=5, \quad\left(1-P_{0}\right)$ corresponding to a $\left(\mu / \lambda N_{s}\right)$ value of 0.7 is 0.85 as seen from figure 5. The true value of availability is thus $59.5 \%$. On the other hand, if $N_{s}=25,\left(1-P_{0}\right)$ corresponding to a $\left(\mu / \lambda N_{s}\right)$ value of 0.7 is 0.965 as seen from figure 5 , giving a true availability of $67.6 \%$.

This example clearly shows the effect of fleet size on availability. Fleet size also affects the repair-depot utilisation. $\left(1-P_{0}\right)$ values close to unity imply that the repairdepot is fully utilised. The depot utilisation and availability are, as is natural, conflicting requirements for small fleet sizes.

\section{Simulation studies for queuing models}

Simulation is a powerful tool of systems engineering methodology which can be profitably used along with analytical models for efficient planning and operation of complex systems. We introduce simulation in our present studies for the following reasons. 
(i) The mathematical modelling of queuing processes becomes too complex for analysis when realistic assumptions regarding the system behaviour are made. While the $M / M / 1$ situation can readily be solved analytically for the general case of arbitrary arrival and service time distributions, service in several phases, variable number of servers etc., analytical solutions become difficult because the processes become non-Markovian (White 1975).

(ii) Even for the simple $M / M / 1$ cyclic queue process, a complete analytical solution for all the relevant quantities of interest is not available. For example, it has been observed only recently (Meyer 1977), that Little's formula connecting queue length and waiting time is not valid for cyclic queuing situations. When it is difficult to obtain analytical results. simulation is often the only technique available to determine the quantity of interest.

(iii) A situation for which most of the analytical results in queues are available is the steady-state condition. It has often been pointed out in the literature and also in the discussion of the present problem in $\S 4$ that, in practice, the behaviour of the system in the transient period is also of great interest as it takes considerable time for a system to settle down to steady-state. Simulation provides a way of studying such behaviour.

(iv) Simulation is often the only available aid in the planning of flying-bases and repair-depots in fleet management which takes into account possible variation in operation and future need. One cannot wait till operating experience is gained and data acquired, as setting up these facilities involves considerable expenditure and time.

The first step in simulation is developing a good simulation model but this is an expensive and time-consuming task. Simulation experiments with such a model can, inter alia, yield the following results:

(i) Choice of analytical models.

(ii) Justification and validation of assumptions made in the analytical model.

(iii) Sensitivity analysis, determining which of the many factors are really significant in affecting the overall system performance.

(iv) Obtaining confidence limits for the performance measures of interest.

(v) Evaluation of the system: for various fleet sizes, repair-depot capacities, arrival and service distributions, the characteristics of system performance such as availability, queue length, utilisation of the repair-depot and waiting time of an aircraft at the repair-depot may be obtained.

(vi) Study of the after-effects of abnormalities like teething troubles and initial advantages.

In this section, we describe simulation results obtained for a single flying-base, repair-depot system. The flow chart of the system is in figure 6 . The simulation was done using GPSS/360 on the IBM $370 / 155$ computer system at the Indian Institute of Technology, Madras. Since simulation is essentially a statistical experiment, to have meaningful conclusions from simulation, it is necessary to make statistically valid statements about the outcome of experiments. In this study, we employ the recently developed regenerative technique (Michael \& Lemoine 1976) to obtain confidence intervals regarding the estimates of the waiting time of an aircraft at the repair-depot. 


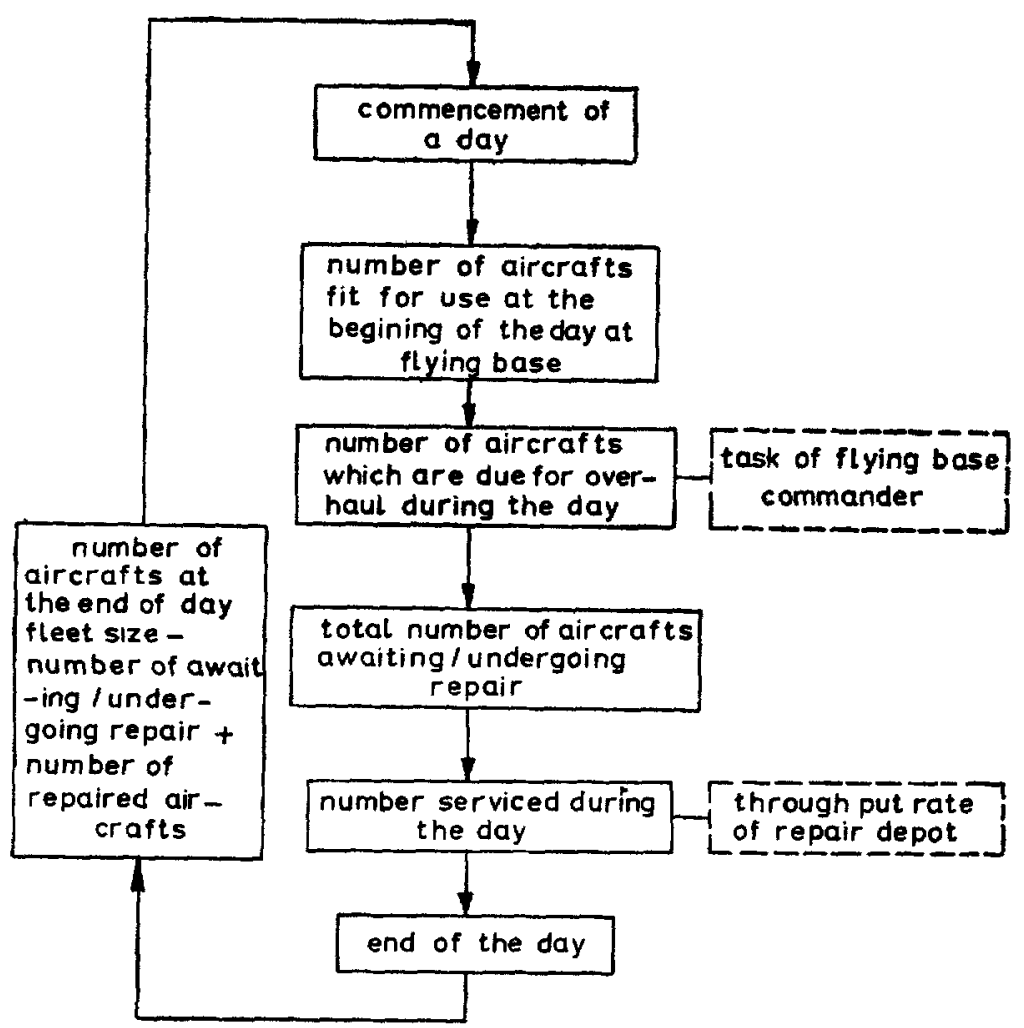

Figure 6. Conceptual flow diagram for flying-base, repair-depot model.

Table 4. Availability for various fleet sizes

Simulation run length $=1400$ days; arrival and service exponential

\begin{tabular}{cccccccccc}
\hline $\begin{array}{c}\text { Fleet size } \\
\text { (availability } \\
\text { \%) in }\end{array}$ & 10 & 20 & 30 & $37 \cdot 5$ & 50 & 60 & 75 & 100 \\
\hline & 10 & 16 & 40 & 72 & 76 & 76 & 80 & 80 & 88 \\
25 & 14 & 44 & 42 & 46 & 58 & 66 & 90 & 78 \\
90 & 10 & 13 & 25 & 27 & 44 & 48 & 54 & 66 \\
100 & &
\end{tabular}

The cyclic queue simulation program using GPSS has 13 blocks for each simulationoriented set of instructions. The total number of blocks required for simulating several $(\mu / \lambda)$ values and fleet sizes is about 200 . The total computer time for one complete set of values is $3 \mathrm{~min}$ and $42 \mathrm{~s}$ for a simulation run length of 1400 days. This simulation run length was chosen on the basis of reaching the steady-state (Ramchand 1977). Table 4 shows the simulation results for various fleet sizes with $M / M / 1$ assumption.

The single-server assumption of the cyclic queue model needs justification. The simulation was repeated with four phases of service and the service time at each phase is described by a random variable $K_{\mathfrak{f}}$ of known distribution. The total service time

$$
K=\sum_{i=1}^{4} K_{i}
$$


Table 5. Availability for various fleet sizes

Simulation run length $=1400$ days. Arrival $=$ Gaussian; service $=$ exponential

\begin{tabular}{ccccccccc}
\hline $\begin{array}{c}\text { Fleet size } \\
\text { (availability } \\
\text { in \%) }\end{array}$ & 10 & 20 & 30 & $37 \cdot 5$ & 50 & 60 & 75 & 100 \\
\cline { 2 - 8 } & 28 & 44 & 60 & 84 & 92 & 92 & 96 & 96 \\
25 & 20 & 36 & 38 & 58 & 64 & 86 & 92 & 96 \\
50 & 8 & 15 & 26 & 31 & 45 & 50 & 59 & 96 \\
100 & & & & & &
\end{tabular}

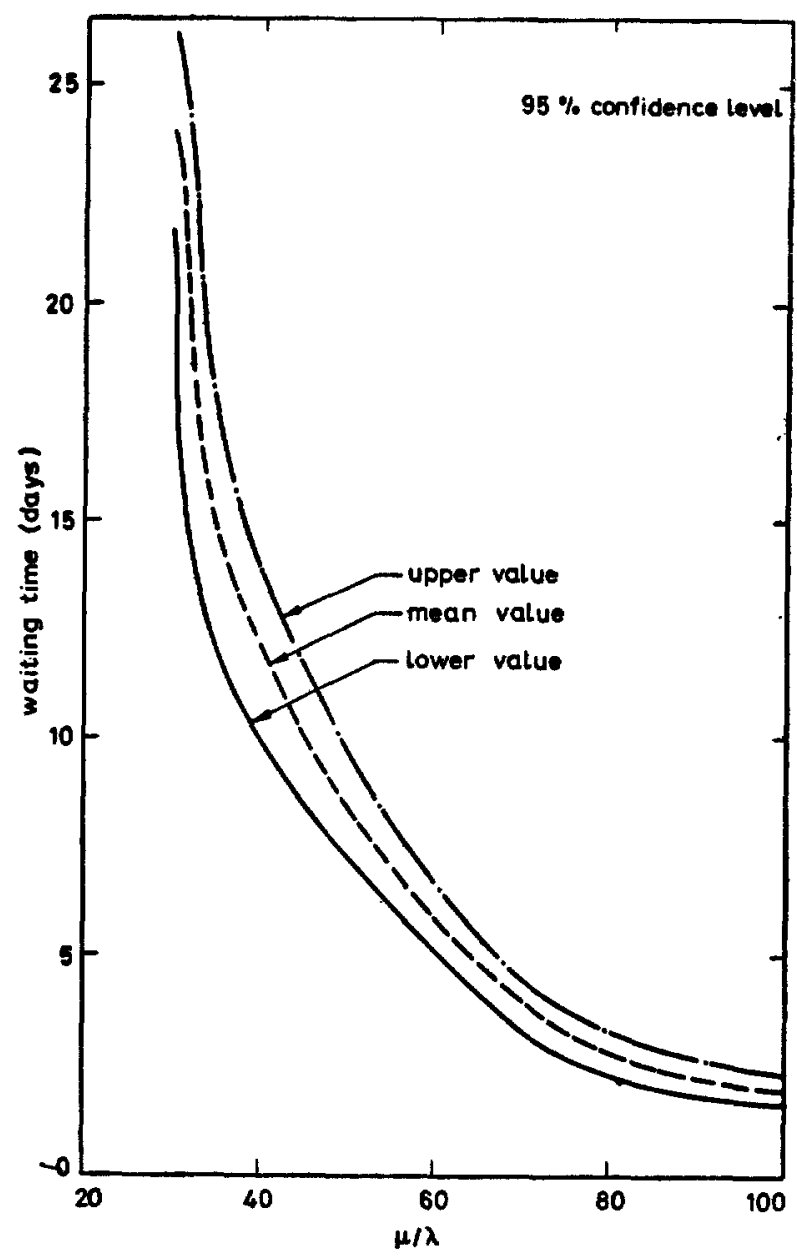

Figure 7. $95 \%$ confidence level for waiting times.

The results are compared with those obtained with equivalent single server assumption. The equivalence is based on identical mean throughput rate of the repair-depot. For the single-server assumption for $\mu / \lambda=30$ and $N_{s}=25$, every 14.27 days on the average an overhauled aircraft was leaving the repair-depot. The corresponding figure for the multiphase case is $\mathbf{1 5 . 7 1}$ days. This shows that the single-server 
assumption gives slightly optimistic estimates of availability. Table 5 shows the availabilities for various fleet sizes and values for Gaussian distributed inter-arrival and service times.

Another interesting result of our simulation studies is the expected waiting time of an aircraft in the repair-depot before it is serviced. This parameter is of considerable interest in optimisation studies and cannot be determined simply, as Little's formula is not valid for cyclic queue situations (Ramchand 1977). Confidence limits for waiting time have been obtained by regenerative techniques (Lavenberg \& Slutz 1975; Michael \& Lemoine 1976). Figure 7 shows the simulation results.

\section{Conclusions}

We may now summarise the significant conclusions of the current study. The availability problem in a single flying-base and repair-depot combination has been modelled and solved. It is shown that an equivalent single-server cyclic queue process provides an adequate analytical description of the problem. This analysis also leads to an elegant definition of the 'smallness' of fleet size in terms of the important traffic parameter of planned service rate of the repair-depot/planned maximum arrival rate from the flying-base.

The simulation package developed using GPSS provides validation of assumptions in analytical models, sensitivity to distributional assumptions and parameters such as queue length and waiting time of an aircraft; it also provides a procedure for estimating confidence intervals for the parameters of interest.

This paper presents only the first stage of a systems engineering study. At the second stage of such a study, one has to consider optimisation problems which lead to minimum cost combinations of fleet size and repair-depot capability to achieve prescribed availability. Ramchand (1977) considers several promising approaches based on dynamic programming and optimal control theory. There are also several possible directions for further work.

There are two general approaches to the analysis of the behaviour of a complex system which is subject to failure: (i) renewal theoretic approach and (ii) queuing theoretic approach. The former approach starts from the distribution of failure times and repair times of a unit and obtains the effects of maintenance strategies using renewal theoretic arguments. Narasimha (1977) uses this approach to study the airworthiness of a certain aircraft in fleet service. In contrast, the queuing theory, as seen from the present work, concentrates on the interaction of an aircraft fieet and its repair facilities, and studies its effect on parameters such as availability. An integration of both the approaches seems to hold great promise in the planning of maintenance policies.

The authors wish to thank Mr V Mani for his help in the GPSS simulation reported in this work and the reviewers for many constructive suggestions. 


\section{List of symbols}

\begin{tabular}{|c|c|}
\hline$A$ & availability (subscript indicates model number) \\
\hline$F$ & flight time per calendar time for the fleet \\
\hline$f$ & actual flight time of an aircraft between overhauls \\
\hline $\boldsymbol{H}$ & mean tour length in which an aircraft logs flight time $f$ \\
\hline$K$ & mean repair time of an individual aircraft at the repair-depot \\
\hline$M$ & total number of critical repairable spares of one type \\
\hline$N_{a}$ & total number of aircraft in the fleet \\
\hline$N_{B}, N_{D}$ & number of aircraft at the flying-base and repair-depot respectively \\
\hline$N_{s}$ & effective fleet size for the single-server cyclic queue model \\
\hline$P_{n}$ & $\begin{array}{l}\text { steady-state probability for } n \text { aircraft being in the queue at the repair- } \\
\text { depot }\end{array}$ \\
\hline$P_{n}(t)$ & $\begin{array}{l}\text { the probability of } n \text { aircraft being in the queue at the repair-depot at } \\
\text { time } t\end{array}$ \\
\hline$q_{n}$ & steady-state probability of $n$ spares being in the repair shop \\
\hline$q_{n}(t)$ & probability of $n$ spares being in the spares repair shop at time $t$ \\
\hline Qss & steady-state number in the queue at repair-depot \\
\hline$Q(t)$ & mean number in the queue at time $t$ at repair depot \\
\hline$T$ & mean calendar time between overhauls i.e. mean tour length \\
\hline$W$ & $\begin{array}{l}\text { mean waiting time, in days, of an aircraft in the repair-depot (includes } \\
\text { servicing time) }\end{array}$ \\
\hline$x|y| z$ & $\begin{array}{l}\text { classification scheme for a queuing system where } x \text { denotes arrival distri- } \\
\text { bution, } y \text { denotes service time distribution and } z \text { the number of servers }\end{array}$ \\
\hline year & one calendar year, equivalent to three hundred working days \\
\hline$\lambda$ & mean arrival rate to the repair-depot $(=1 / T$ in flow model $)$ \\
\hline$\mu$ & mean service rate of the repair-depot \\
\hline$\mu_{R}$ & mean service rate of the repairable spares at the spares repair shop \\
\hline
\end{tabular}

\section{References}

Agnew C E 1976 Oper. Res. 34400

Chittaranjan N 1974 Proceedings of management seminar on effective maintenance (Poona: Tata Management Centre)

Dade M 1973 RAND Report R-1299 PR, RAND Corporation, Santa Monica, California, USA

Donaldson T S 1971 RAND Report R-755-PR, RAND Corporation, Santa Monica, California, USA

Geisler M A 1960 Manage. Sci. 6444

Gross D, Kahn H D \& Marsh J D 1975 The George Washington University Report No. T-322

Koenigsberg E 1960 Oper. Res. 8246

Koopman B O 1972 Oper. Res. 201089

LaValle R S \& Stoller D S 1962 IRE Trans. Reliab. Qual. Control RQC-11 28

Lavenberg G S \& Slutz D R 1975 IBM J. Res. Dev. 19458

Little J D C 1961 Oper. Res. 9383

Maher M J \& Cabrera J G 1975 Oper. Res. Q. 24603 
Meyer K H F 1977 Lecture notes in economics and mathematical systems (New York: SpringerVerlag) p. 31

Michael AV \& Lemoine A J 1976 Proceedings of the Winter Simulation Conference, ed. H J Highland (New York: State Univ. Agr. and Tech.) p. 41

Moe T A 1976 IEEE Trans. Reliab. R25 243

Narasimha R 1977 J. Franklin Inst. 30315

Narayan Bhat U 1969 Manage. Sci. 15 B280

Ramchand K 1977 Systems engineering approach to air-fleet and maintenance planning, Ph.D. thesis, Department of Aeronautical Engineering, Indian Institute of Science, Bangalore

Rose M 1973 Nav. Res. Log. Q. 20147

Sarma V V S, Ramchand K \& Rao A K 1977 IEEE Trans. Reliab. R24 253

Schriber T J 1974 Simulation using GPSS (New York: John Wiley)

Shannon R E 1976 Proceedings of the Winter Simulation Conference, ed. H J Highland (New York: State Univ. Agr. and Tech.) 19

White J A, Schmidt J W \& Bennett G K 1975 Analysis of queueing systems (New York: Academic Press)

Wood J K R 1971 Proceedings of NATO Conference ed. D Grouchko (New York: Gordon and Breach) p. 145 